\title{
FIXED POINTS IN NONCONVEX DOMAINS
}

\author{
ERIC CHANDLER AND GARY FAULKNER
}

\begin{abstract}
A lemma of Janos is used to prove that nonexpansive self-maps which "shrink" a compact set $X$ away from its boundary in $\overline{c o} X$ have fixed points in $X$. The lemma is further employed to derive a version, for nonexpansive maps on star-shaped sets, of Janos and Solomon's fixed-point theorem for continuous maps on spaces having an attractor for compact sets.
\end{abstract}

Janos [2], using a result of A. D. Wallace [4, Theorem 1] in the theory of topological semigroups arrived at the following lemma.

LEMMA 1 (JANOS [2]). Let $(X, d)$ be a compact metric space and $f: X \rightarrow X$ with $\left\{f^{n} \mid n>1\right\}$ equicontinuous. Then there exists a retraction $r: X \rightarrow C_{f}$ where $C_{f}$ denotes the core of $f$; i.e. $C_{f}=\bigcap\left\{f^{n}(X) \mid n>1\right\}$.

A particularly significant feature in the proof of this lemma is the demonstration that the retraction $r$ of $X$ onto $C_{f}$ is in the closure, in the pointwise topology, of the set $\Gamma_{n}=\left\{f^{m} \mid m>n\right\}$ for each $n>1$ (cf. [2] and [3]). This enables us to state a version of the lemma for nonexpansive maps.

LEMMA 2. Let $T$ be a nonexpansive self-map of a compact metric space $(X, d)$. Then there exists a nonexpansive retraction $r: X \rightarrow C_{T}$ where

$$
C_{T}=\cap\left\{T^{n}(X) \mid n>1\right\} .
$$

Proof. The family $\left\{T^{n}\right\}$ is equicontinuous, for given any $\varepsilon>0$ if we merely choose $\delta=\varepsilon$ then $d(x, y)<\delta$ implies that $d\left(T^{n} x, T^{n} y\right)<d(x, y)<\varepsilon$ for all $n>1$. Now the retraction $r$ guaranteed by Lemma 1 is the pointwise limit of mappings of the form $T^{n}$. Thus, for any $x, y \in X$ and any $\varepsilon>0$ there exists an $n$ such that $d\left(r x, T^{n} x\right)<\varepsilon$ and $d\left(T^{n} y, r y\right)<\varepsilon$. Hence

$$
\begin{aligned}
d(r x, r y) & <d\left(r x, T^{n} x\right)+d\left(T^{n} x, T^{n} y\right)+d\left(T^{n} y, r y\right) \\
& <d\left(T^{n} x, T^{n} y\right)+2 \varepsilon<d(x, y)+2 \varepsilon .
\end{aligned}
$$

Since $\varepsilon$ was arbitrary this shows $d(r x, r y)<d(x, y)$ and thus $r$ is a nonexpansive mapping.

A normed linear space $X$ is said to be strictly convex if whenever $x, y \in X$ with $x \neq y,\|x\|=\|y\|=S$ and $0<\alpha<1$, then $\|\alpha x+(1-\alpha) y\|<S$. A useful property of strictly convex spaces is that for any three noncollinear points $x, y, z \in X$, the triangle inequality is strict; i.e. $d(x, z)<d(x, y)+d(y, z)$.

Received by the editors January 21, 1980 and, in revised form, March 12, 1980.

1980 Mathematics Subject Classification. Primary 47H10.

Key words and phrases. Fixed points, nonexpansive mappings, retractions, strictly convex spaces. 
In the results which follow, we shall denote by $\partial^{\prime} X$ the boundary of $X$ in the closed convex hull of $X$.

THEOREM 1. Let $X$ be a compact subset of a strictly convex normed linear space $E$ and let $T: X \rightarrow X$ be nonexpansive. If there exists an $n>1$ such that $T^{n} X \cap \partial^{\prime} X=$ $\varnothing$ then $T$ has a fixed point in $X$.

Proof. As before let $C_{T}=\cap{ }_{n=1}^{\infty} T^{n} X$. By Lemma 2 there exists a nonexpansive retraction $r: X \rightarrow C_{T}$. Suppose $C_{T}$ is not convex. Then there exists $x, y \in C_{T}$ such that $\overline{x y}=\{\alpha x+(1-\alpha) y \mid 0<\alpha \leqslant 1\}$ is not contained entirely in $C_{T}$. Let $a=$ $\sup \left\{\beta \mid \alpha x+(1-\alpha) y \in C_{T}, 0 \leqslant \alpha \leqslant \beta\right\}$. Since $C_{T}$ is compact, $z=a x+$ $(1-a) y$ is in $C_{T}$. Now if $x y$ contains no points of $X \backslash C_{T}$ then $z$ is a $\partial^{\prime}$-boundary point of $X$ in $C_{T} \subseteq T^{n} X$ contradicting the hypothesis. Hence there exists a point $w \in \overline{x y} \cap\left\{X \backslash C_{T}\right\}$. But then we shall have

$$
\|r x-r w\|+\|r w-r y\| \leqslant\|x-w\|+\|w-y\|=\|x-y\|
$$

and, since $r x=x, r y=y$,

$$
\|x-r w\|+\|r w-y\|<\|x-y\| .
$$

But $r w \in C_{T}$ and so $w \neq r w$. The mapping $r$ is nonexpansive and so $r w$ is not collinear with $x, y$ for if $w=b x+(1-b) y$ and $r w=c x+(1-c) y$ we shall have $b \neq c$, say, $c<b<1$. But then

$$
\|x-w\|=(1-b)\|x-y\|
$$

and

$$
\|r x-r w\|=\|x-r w\|=(1-c)\|x-y\|
$$

and since $(1-c)>(1-b)$ we would have $\|x-w\|<\|r x-r w\|$, a contradiction. If, on the other hand, $0<b<c$ then $\|y-w\|=b\|x-y\|$ and $\|r y-r w\|=$ $\|y-r w\|=c\|x-y\|$ and so $\|y-w\|<\|r y-r w\|$, another contradiction. Thus, $x, y, r w$ are not collinear. But then $\|x-r w\|+\|r w-y\|>\|x-y\|$ contradicting an earlier inequality. Thus $C_{T}$ must be convex. Now $T: C_{T} \rightarrow C_{T}$ and so $T$ has a fixed point in $C_{T}$ by Schauder's Theorem.

COROLlary 1. Let $X$ be a compact subset of a strictly convex normed linear space $E$ and suppose $T, g$ are self-maps of $X$ such that $g$ is continuous, $T$ is nonexpansive and $T^{n} X \cap \partial^{\prime} X=\varnothing$ for some $n \geqslant 1$. If $T g=g T$ then $T, g$ have a common fixed point in $X$.

COROllary 2. Let $X$ be a compact subset of a strictly convex normed linear space $E$. Suppose that $\left\{T_{\alpha}\right\}$ is a family of commuting, nonexpansive self-maps of $X$ and that for one $T \in\left\{T_{\alpha}\right\}$, there exists an $n \geqslant 1$ such that $T^{n} X \cap \partial^{\prime} X=\varnothing$. Then the family $\left\{T_{\alpha}\right\}$ has a common fixed point in $X$.

The proofs of these corollaries utilize standard techniques.

The suspicion that "compact" may not be replaced by "closed and bounded" in the hypotheses of Theorem 1 is easily confirmed by the following example in the Hilbert space $l_{2}$ : Let $e_{n}$ be the $n$th unit vector in $l_{2}$ and let 


$$
X=\bigcup_{n=1}^{\infty} B\left(e_{n}, n /(2 n+1)\right)
$$

Then $X$ is closed and bounded. Define the mapping $T: X \rightarrow X$ by $T\left(x_{1}, x_{2}, x_{3}, \ldots\right)=\left(0, x_{1}, x_{2}, x_{3}, \ldots\right)$. Then $T$ is nonexpansive and $T X \cap \partial^{\prime} X=$ $\varnothing$. But $T$ has no fixed point in $X$.

Definition. Let $X$ be a topological space and $f: X \rightarrow X$ a map. A nonempty subset $M \subseteq X$ is called an "attractor for compact sets under $f$ " if (a) $M$ is compact and $f M \subseteq M$, (b) for any compact set $G \subseteq X$ and open set 0 containing $M$, there exists an $N=N(G, 0)$ such that $f^{n} G \subseteq 0$ for all $n>N$.

Janos and Solomon [3], using Lemma 1, have proven

Theorem 2. Let $f: G \rightarrow G, G$ a closed, convex subset of a Banach space, $f$ a mapping satisfying:

(i) there exists $M \subseteq G$ an attractor for compact sets under $f$, and

(ii) the family $\left\{f^{n}\right\}_{n=1}^{\infty}$ is equicontinuous.

Then $f$ has a fixed point in $X$.

We shall prove a result analogous to this theorem for nonexpansive maps on star-shaped sets. The proof parallels that of Janos and Solomon.

Theorem 3. Let $S$ be a closed, star-shaped subset of a Banach space $E$ and $T$ : $S \rightarrow S$ nonexpansive. If there exists $M \subseteq S$, an attractor for compact sets, then $T$ has a fixed point in $S$.

Proof. Let $p$ be a star-point of $S$ and define the closed star-hull of $M$ to be $\operatorname{sh} M=\operatorname{cl}\{t p+(1-t) x \mid 0 \leqslant t<1, x \in M\}$. $M$ is compact, so $M \cup\{p\}$ is compact. Now the convex hull of a compact set is compact, so $\overline{c o}[M \cup\{p\}]$ is compact. But $\operatorname{sh} M \subseteq \overline{c o}[M \cup\{p\}]$ so $s \bar{h} M$ is compact. Let

$$
X=\operatorname{cl}\left\{\cup T^{n}(\operatorname{sh} M) \mid n \geqslant 0\right\} .
$$

Since $T\left[T^{n}(\operatorname{sh} M)\right]=T^{n+1}(\operatorname{sh} M)$ for all $n \geqslant 0$ and $T$ is continuous, $T: X \rightarrow X . X$ is compact, for if $0_{\varepsilon / 2}$ is the $\varepsilon / 2$-parallel set of $M$ (i.e. $0_{\varepsilon / 2}=\cup_{x \in M} S(x, \varepsilon / 2)$ ) there exists an $N$ such that $n>N$ implies $T^{n}(\mathrm{~s} \overline{\mathrm{h}} M) \in 0_{\varepsilon / 2}$. But $M$ compact implies that $M$ has a finite $\varepsilon / 2$-mesh. Thus $0_{\varepsilon / 2}$ has a finite $\varepsilon$-mesh. So $\cup_{n>N} T^{n}(\operatorname{sh} M)$ has a finite $\varepsilon$-mesh and since $\cup_{n<N} T^{n}(\operatorname{sh} M)$ is compact, $X$ is itself compact.

Now let $C_{T}=\bigcap_{n=0}^{\infty} T^{n}(X) . C_{T}$ is nonempty and compact.

$$
T C_{T}=T \bigcap_{n=0}^{\infty} T^{n}(X)=\bigcap_{n=0}^{\infty} T^{n+1}(X)=C_{T}
$$

so by the definition of $M, C_{T} \subseteq M$. By Lemma 2 there exists a nonexpansive retraction $r: X \rightarrow C_{T}$. The map $\operatorname{Tr}: \operatorname{sh} M \rightarrow C_{T}$ is a nonexpansive self-map of a compact star-shaped set $\mathrm{sh} M$ and so by Dotson's theorem [1] has a fixed point $x \in \overline{s h} M$. Now $r(x) \in C_{T}$ and $T: C_{T} \rightarrow C_{T}$ so $x=\operatorname{Tr}(x) \in C_{T}$. But then $r(x)=x$ and thus $T x=x$. 


\section{REFERENCES}

1. W. G. Dotson, Jr., Fixed-point theorems for non-expansive mappings on star-shaped subsets of Banach spaces, J. London Math. Soc. 2 (1972), 408-410.

2. L. Janos, On representations of self mappings, Proc. Amer. Math. Soc. 26 (1970), 529-533.

3. L. Janos and J. L. Solomon, A fixed-point theorem and attractors, Proc. Amer. Math. Soc. 71 (1978), 257-261.

4. A. D. Wallace, Inverses in Euclidean mobs, Math. J. Okayama Univ. 3 (1953), 23-28.

Departamat of Mathematics, North Carolna State University, Ralbigh, North Carolna 27650 